\title{
Lung mucosal immunity: immunoglobulin-A revisited
}

\section{To the Editor:}

We read through the Review by Pilette et al. [1] with extreme interest and would like to add a contribution to the subject of airways immunity in chronic obstructive pulmonary disease (COPD). For a long time, we have witnessed, at least in Italy, the predominance of the notion that all COPD patients have a certain degree of immune deficiency as a basic pathogenetic mechanism, since they experience recurrent bronchitis exacerbations. Scientific evidence of true general or local immune defects in COPD is, in our opinion, inconclusive, although we fully agree on the point made by the authors that all the studies in the literature share methodological limitations, both in sampling and analysis techniques and in selection of patients. In our experience, different immune components can appear either similar to controls or increased (as a likely consequence of repeated stimulation by exacerbations or chronic bacterial colonization of the airways), or decreased in a specific group of COPD patients. Indeed, we recently reported decreased numbers of $\mathrm{CD} 3$ and CD8 lymphocytes in the bronchial biopsies of severe COPD patients, associated with an increase of neutrophils and macrophages [2]. With particular regard to immunoglobulin (Ig)-A, we found only an insignificant increase in patients with mild COPD who had never smoked [3], and a high increase in severe but clinically stable COPD patients with chronic tracheostomy and a high level of bacterial colonization [4]. The experience with tracheostomized COPD patients is very interesting because they provide a model of bacteria/host interaction in which the role of immunity can be evaluated prospectively. The presence of high levels of $\operatorname{IgA}$ in bronchial aspirates could, in part, justify the relatively low rate of lower respiratory tract infections in these patients after discharge from hospital [5].

We appreciated the long and accurate list of defence mechanisms in the respiratory tract reported by the authors, which clearly shows that specific immune functions are only a part of the airways protection system. Indirect proof that immunoglobulins are only a part, albeit an important part, of mucosal immunity, comes from studies on the stimulation of mucosaassociated lymphoid tissues with oral vaccines or bacterial extracts, which have been demonstrated to reduce the impact of bronchitis exacerbations, at least in mild-to-moderate chronic obstructive pulmonary disease [6]. The mechanisms of action were reflected in an increase of immunoglobulin-A in the airways' fluids, but more solid evidence, from a biological point of view, was the activation of alveolar macrophages [7]. In our opinion, the potential of oral bacterial extracts to stimulate immunoglobulin-A production in the airways should be further investigated. In conclusion, we would once again like to stress that the impairment of defence systems in the airways is not simply a question of specific immunity, and that a clinically relevant imbalance in chronic obstructive pulmonary disease must be evaluated within a complex defence network concept, rather than in a simple cause/effect perspective.

\section{Lusuardi, A. Capelli, A. Di Stefano, C.F. Donner}

Salvatore Maugeri Foundation, IRCCS, Scientific Institute of Rehabilitation, Division of Pulmonary Disease, Veruno, NO, Italy.

\section{References}

1. Pilette C, Ouadrhiri Y, Godding V, Vaerman J-P, Sibille Y. Lung mucosal immunity: immunoglobulinA revisited. Eur Respir $J$ 2001; 18: 571-588.

2. Di Stefano A, Capelli A, Lusuardi M, et al. Decreased $\mathrm{T}$ lymphocyte infiltration in bronchial biopsies of subjects with severe chronic obstructive pulmonary disease. Clin Exp Allergy 2001; 31: 893-902.

3. Lusuardi M, Capelli A, Cerutti CG, Spada EL, Donner CF. Airways inflammation in subjects with chronic bronchitis who have never smoked. Thorax 1994; 49: 1211-1216.

4. Lusuardi M, Capelli A, Cerutti CG, Gnemmi I, Zaccaria S, Donner CF. Influence of clinical history on airways bacterial colonization in subjects with chronic tracheostomy. Respir Med 2000; 94: 436-440.

5. Harlid R, Andersson G, Frostell CG, Jorbeck HJA, Ortqvist AB. Respiratory tract colonization and infection in patients with chronic tracheostomy. A one-year study in patients living at home. Am J Respir Crit Care Med 1996; 154: 124-129.

6. Bergemann R, Brandt A, Zoellner U, Donner CF. Preventive treatment of chronic bronchitis: a metaanalysis of clinical trials with a bacterial extract (OM$85 \mathrm{BV}$ ) and a cost-effectiveness analysis. Monaldi Arch Chest Dis 1994; 49: 302-307.

7. Lusuardi M, Capelli A, Carli S, Spada EL, Spinazzi A, Donner CF. Local airways immune modifications induced by oral bacterial extracts in chronic bronchitis. Chest 1993; 103: 1783-1791.

\section{From the authors:}

We thank M. Lusuardi and colleagues for their comments on our review [1] and more specifically for the discussion related to airways immunity in chronic obstructive pulmonary disease (COPD). 
As we already stated in our review [1], and as expanded by M. Lusuardi and colleagues, the observations on the immune and inflammatory reaction in the airways of COPD patients vary considerably in the literature and therefore, their clinical correlates are so far hypothetical. We certainly did not intend to suggest that COPD results only from an impairment of "specific immunity". Thus, while the contribution of inflammatory and immune processes to the airways remodelling observed in COPD remains speculative, it is likely that this remodelling has a profound impact on the most important first-line defence of the respiratory tract, namely the mucociliary system.

In this context, the observation that the epithelial content of secretory piece (the active carrier of immunoglobulin (Ig)-A and IgM to mucosal surfaces) is dramatically decreased in airways from severe COPD, illustrates an interesting link between bronchial structure changes and the immune response (both innate and specific, since $\operatorname{IgA}$ and IgM participate in both responses).

Moreover, although increased IgA levels in bronchial secretions could be considered beneficial, it remains to be demonstrated that a substantial part of this $\operatorname{IgA}$ is able to efficiently neutralize bacterial or viral particles. In addition, the interaction of IgA with the phagocytic system, particularly with neutrophils and alveolar macrophages, could modulate the oxidative burst and cytokine release with potential implications on the defence against pathogens as well as on bronchial and lung damage.

In conclusion, mucosal immunity takes part in lung homeostasis and respiratory disorders. To what extent secretory immunity is integrated into the systemic response and influences the outcome of lung disorders and vice versa remains largely uncertain.

\section{Pilette, Y. Sibille}

Unit of Experimental Medicine, Christian de Duve Institute of Cellular Pathology, University of Louvain, Brussels, Belgium.

\section{References}

1. Pilette C, Ouadrhiri Y, Godding V, Vaerman J-P, Sibille Y. Lung mucosal immunity: immunoglobulin-A revisited. Eur Respir J 2001; 18: 571-588. 